\title{
Simvastatin-Induced Rhabdomyolysis and Acute Renal Injury
}

\author{
Abdelkarim Waness $^{\text {a }}$ Sami Bahlas $^{\mathrm{b}}$ Saad Al Shohaib ${ }^{c}$ \\ ${ }^{a}$ Department of Internal Medicine, King Abdulaziz Medical City, Riyadh, and Departments of ${ }^{b}$ Medicine and \\ 'Nephrology, King Abdulaziz University, Jeddah, Saudi Arabia
}

\section{Key Words}

Simvastatin $\cdot$ Rhabdomyolysis · Acute renal failure $\cdot$ Hemodialysis $\cdot$ Diabetes mellitus

\begin{abstract}
Simvastatin is one of the most commonly prescribed CoA reductase inhibitors. The safety profile of this drug has been widely discussed in the medical and consumer advocacy communities. Like other statins, simvastatin can cause a serious and potentially life-threatening complication: rhabdomyolysis. We describe a case of simvastatin-induced rhabdomyolysis complicated by acute renal failure requiring urgent hemodialysis. The relative safety of simvastatin compared to other HMG-CoA reductase inhibitors and the conditions that can potentiate its toxicity are discussed. The clinical features of rhabdomyolysis, and subsequent acute renal failure, and their treatment modalities are presented.
\end{abstract}

Copyright $\odot 2008$ S. Karger AG, Basel

\section{Introduction}

HMG-CoA reductase inhibitors (statins) are the cornerstone of modern therapy for dyslipidemias and ischemic heart disease. Since the approval of the first one, lovastatin $\left(\right.$ Mevacor ${ }^{\circledR}$ ), in 1987 [1], statins have been increasing in number, effectiveness and market share. This evolution, however, brought with it a number of complications and controversies. Among the most worrisome

\section{KARGER}

Fax +41613061234

E-Mail karger@karger.ch

www.karger.com
(C) 2008 S. Karger AG, Basel

0253-5068/08/0264-0394\$24.50/0

Accessible online at:

www.karger.com/bpu complications of statins are the increase in liver transaminases level and myopathy. The latter can range from simple diffuse muscle soreness to a more severe, and even fatal, rhabdomyolysis.

Rhabdomyolysis is defined by the breakdown of striated muscle fibers with release of toxic intracellular components into the systemic circulation. Beside the clinical picture of severe diffuse muscular pain, rhabdomyolysis is diagnosed with tenfold elevation or more in the serum creatine kinase (CK) level. Many other hemodynamic and metabolic derangements might follow this muscular injury. Hypovolemia, fluid sequestration, hyperkalemia, cardiac arrhythmia, hypocalcemia, metabolic acidosis, disseminated intravascular coagulation, acute renal failure, and other disturbances can occur with rhabdomyolysis [2]. Many predisposing factors for rhabdomyolysis have been identified: some of them are well known like female gender, small body frame, elderly people, presence of multiple co-morbidities, perioperative periods, crush injury, overexertion, seizures, alcohol abuse, certain medications, and some industrial toxins $[3,4]$. Other etiologies are less known or rare, e.g. fire ant bites [5], pomegranate juice [6] and lightning [7].

All statins are well known to cause a variable degree of myopathies, from simple muscular soreness to more serious myositis and even fatal rhabdomyolysis. This dreaded complication is worse when any statin is combined with other medications like fibric acid derivatives, niacin, amiodarone, cyclosporine, macrolides, azole antifungal agents, diltiazem, verapamil, HIV protease inhibitors, 
etc. There are medical conditions that predispose to muscular statin toxicity, like diabetes mellitus, renal impairment, familial muscular disorders, hypothyroidism, and the consumption of grapefruit juice [8].

Cervastatin $\left(\mathrm{Baycol}^{\circledR}\right)$ was withdrawn from the US market in August 2001 after many reported cases of severe rhabdomyolysis, acute renal failure and death. Lovastatin, simvastatin and atorvastatin can also cause rhabdomyolysis, but to a much lesser degree [9].

Rosuvastatin $\left(\right.$ Crestor $^{\circledR}$ ) was the last HMG-CoA reductase inhibitor to be approved by the Food and Drug Administration (FDA, USA) in August 2003 [1]. Since the first step of its approval, rosuvastatin has been causing more controversy than other statins. A good number of trials were conducted to look at the safety of rosuvastatin. Many investigators came up with a favorable conclusion, stating rosuvastatin has a similar adverse effect profile compared to other HMG-CoA reductase inhibitors [1015]. Other authors took a more cautious attitude with regard to the spectrum of its possible adverse effects [1619].

The first simvastatin, Zocor ${ }^{\circledR}$, was approved by the FDA in 1991. Since then, it has been one of the most prescribed HMG-CoA reductase inhibitors. In 2005, the total sales for statins were estimated at USD 16 billion. Zocor $^{\circledR}$ ranked No. 2 after atorvastatin [1]. In 2006, the FDA approved generic simvastatin, which competes with Zo$\operatorname{cor}^{\circledR}$ and other products for the lucrative statins market.

In anticipation of the patent expiration of Zocor ${ }^{\circledR}$, Merck, the maker of the drug, launched Vytorin ${ }^{\circledR}$ in 2004. It contains both simvastatin and ezetimibe.

The risk of simvastatin-induced rhabdomyolysis is thought to be low $[20,22]$. Its myotoxicity can occur either as monotherapy [21-23] or in combination therapy [24-27]. Compared to other statins, simvastatin is thought to be in the middle of the statin muscular adverse effect scale. It can potentially cause more rhabdomyolysis than pravastatin and fluvastatin [28]. One study found that simvastatin is more toxic to the muscle than atorvastatin [29].

\section{Case Report}

A 63-year-old female, with multiple medical conditions, presented to King Abdulaziz University Hospital Emergency Room on April 28, 2007, with the chief complaint of severe generalized myalgia. The patient is legally blind and is known to have had a history of type 2 diabetes mellitus for the past 20 years, hypertension for 5 years, dyslipidemia for 1 year, and a poorly documented ischemic heart disease. The patient was taking metformin, aspi- rin, atenolol, perindopril, clopidogrel, furosemide, and simvastatin. Since the patient was new to our institution, it was related that she started taking simvastatin $10 \mathrm{mg}$ daily for the past 1 year. The dose was increased to $40 \mathrm{mg}$ daily, 10 days prior to her initial first visit at our institution. After a few days, she started experiencing fatigue, malaise, poor appetite and vomiting. Subsequently, she developed severe muscle pain in her extremities and abdomen, followed by urine discoloration (red urine noted by relatives) and oliguria (twice per day). She was brought to our Department of Emergency Medicine where she was evaluated and subsequently admitted.

\section{Physical Examination}

On physical examination, the patient was a middle-aged female who was drowsy but arousable, complaining of diffuse pain. BP $137 / 74 \mathrm{mmHg}$; pulse $70 \mathrm{bpm}$; RR 24/min; temperature $36.4^{\circ} \mathrm{C}$; weight $59 \mathrm{~kg}$. She had a $2 / 6$ ejection systolic murmur at the left sternal border, and diffuse abdominal tenderness without guarding or organomegaly. Her neuromuscular examination showed severe muscular tenderness on palpation of the four extremities with diffuse decreased motor strength to $3 / 5$, otherwise there was no focal motor deficit, the cranial nerves were intact, no swelling or erythema of her extremities, and no involvement of her joints. The rest of her examination was unremarkable.

\section{Laboratory Findings on Admission}

A preliminary laboratory evaluation was done after her arrival to the department of Emergency Medicine (April 28, 2007), which shows the following findings: WBC $20.3 \times 10^{3} / \mu \mathrm{l} \times 10^{3}$ (87\% neutrophils); hemoglobin $11.7 \mathrm{~g} / \mathrm{dl}$; hematocrit $33.8 \%$; platelets $332 \times 10^{3} / \mu \mathrm{l}$; sodium $110 \mathrm{mmol} / \mathrm{l}$; potassium $5.8 \mathrm{mmol} / \mathrm{l}$; magnesium $0.88 \mathrm{mmol} / \mathrm{l}$; chloride $7 \mathrm{mmol} / \mathrm{l}$; random glucose 8.0 $\mathrm{mmol} / \mathrm{l}$; BUN $36.3 \mathrm{mmol} / \mathrm{l}$; serum creatinine $384 \mu \mathrm{mol} / \mathrm{l}$; serum calcium $1.72 \mathrm{mmol} / \mathrm{l}$; serum phosphate $2.71 \mathrm{mmol} / \mathrm{l}$; creatine kinase (CK): 31,207 U/1 (CK-MB $320 \mathrm{IU} / \mathrm{l})$; troponin-I $0.07 \mu \mathrm{g} / \mathrm{l}$; lactate dehydrogenase $1,715 \mathrm{U} / \mathrm{l}$; aspartate aminotransferase (AST) $549 \mathrm{U} / \mathrm{l}$; alanine aminotransferase (ALT) $576 \mathrm{U} / \mathrm{l} ; \gamma$-glutamyl transferase (GGT) $284 \mathrm{U} / \mathrm{l}$, and alkaline phosphatase (ALP) $164 \mathrm{U} / \mathrm{l}$.

The rest of her laboratory findings, including thyroid function testing, were unremarkable.

\section{Treatment}

Based on the clinical presentation and laboratory findings, the diagnosis of rhabdomyolysis complicated by acute renal injury was established. Looking back at the fact that her dose of simvastatin was increased from 10 to $40 \mathrm{mg} / \mathrm{day}$, we can conclude that this drug was the most likely etiology behind her rhabdomyolysis. This high probability is further confirmed by the elevation noticed in her liver enzymes, a well-known complication of all statins.

The patient was admitted to the medical ward, simvastatin was stopped and urgent treatment with hemodialysis was started. Her first session was on April 29, 2007, with little clinical improvement; the patient was still oliguric, complaining of diffuse myalgia. Her blood test that day showed mild improvement in the CK level $28,108 \mathrm{IU} / \mathrm{l}$, potassium level $5.5 \mathrm{mmol} / \mathrm{l}$, phosphate level 2.43 $\mathrm{mmol} / \mathrm{l}$, and $\mathrm{WBC}$ to $18.1 \times 10^{3} / \mu \mathrm{l}$. Her serum creatinine level rose, however, to $462 \mu \mathrm{mol} / \mathrm{l}$, and the serum calcium worsened to $1.35 \mathrm{mmol} / \mathrm{l}$. Furthermore, blood and urine cultures were done and were negative. The patient did not receive any antibiotic therapy. 
Fig. 1. Serum creatine kinase levels.

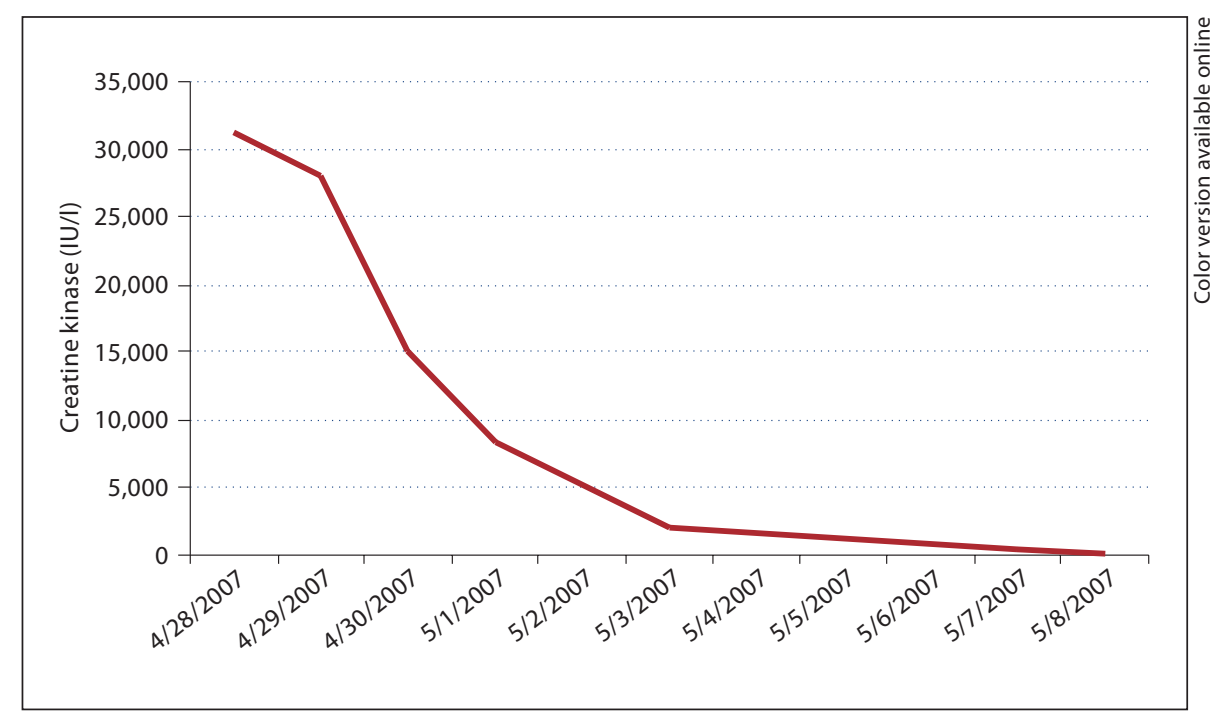

Table 1. Main laboratory findings by date

\begin{tabular}{lllllllllll}
\hline & $4 / 28 / 07$ & $4 / 29 / 07$ & $4 / 30 / 07$ & $5 / 1 / 07$ & $5 / 2 / 07$ & $5 / 3 / 07$ & $5 / 7 / 07$ & $5 / 8 / 07$ & $5 / 12 / 07$ & $5 / 16 / 06$ \\
\hline Creatine kinase & 31,207 & 28,108 & & 8,407 & & 1,975 & & 95 & & \\
Serum creatinine & 384 & 462 & 393 & 264 & 275 & 343 & 703 & & 251 & 171 \\
Potassium & 5.8 & 5.5 & & 3.1 & & 3.6 & 3.7 & & 3.8 & 5.0 \\
Sodium & 110 & 110 & 122 & 125 & 127 & 122 & & 136 & 129 & 134 \\
Calcium & 1.72 & 1.35 & 1.40 & 1.60 & & 1.80 & 1.90 & & & \\
Phosphate & 2.71 & 2.43 & 2.07 & 1.17 & & 1.12 & & & & \\
WBC & 20.9 & 18.1 & & & & & 12.3 & & 11.7 \\
\hline
\end{tabular}

Subsequently, this patient underwent four more hemodialysis sessions on the following days: April 30, May 1, 5, and 7, 2007. She felt progressively better afterwards. Her severe muscle pain resolved and she started having better urine output. Table 1 summarizes her successive important laboratory findings. The serum CK level showed a sharp and steady decline after stopping simvastatin. It completely normalized within 10 days of the patient's admission to our institution (fig. 1). The serum electrolytes, including potassium, sodium, calcium and phosphate, also progressively improved. The kidney function, however, worsened after initial treatment, requiring therefore further hemodialysis. After the fifth and last dialysis episode, the patient was on her way to complete renal function recovery (fig. 2).

\section{Follow-Up}

After a 21-day stay at King Abdulaziz University Hospital, the patient was discharged home, on May 19, 2007, following complete clinical recovery and normalization of her laboratory data. She received the following medications: metformin $500 \mathrm{mg}$ p.o. t.i.d., nifedipine $20 \mathrm{mg}$ p.o. b.i.d., isosorbide dinitrate $10 \mathrm{mg}$ p.o. b.i.d., pantoprazole $40 \mathrm{mg}$ p.o. q.d., with iron and vitamin $\mathrm{D}$ replacements.
The patient was seen at the outpatient clinic on May 30, 2007 for a follow-up appointment. She was doing well overall, denied further muscular pain, and was urinating in a satisfactory fashion. A repeat serum creatinine level was done that day and it was $80 \mu \mathrm{mol} / \mathrm{l}$. She also had a liver function testing done on May 8 , 2007 that showed major improvement with AST $28 \mathrm{U} / \mathrm{l}$ and ALT $100 \mathrm{U} / \mathrm{l}$.

\section{Discussion}

Our 63-year-old female patient, with diabetes mellitus, hypertension, dyslipidemia, and ischemic heart disease, represents a good example of the usual 'bread and butter' of many physician practices around the world. These diabetic, hypertensive, dyslipidemic, vasculopaths are treated by family practitioners, internists, endocrinologists, cardiologists, nephrologists, and even by pharmacists and nurses in some countries. Polypharmacy usually accompanies these co-morbid conditions, which 


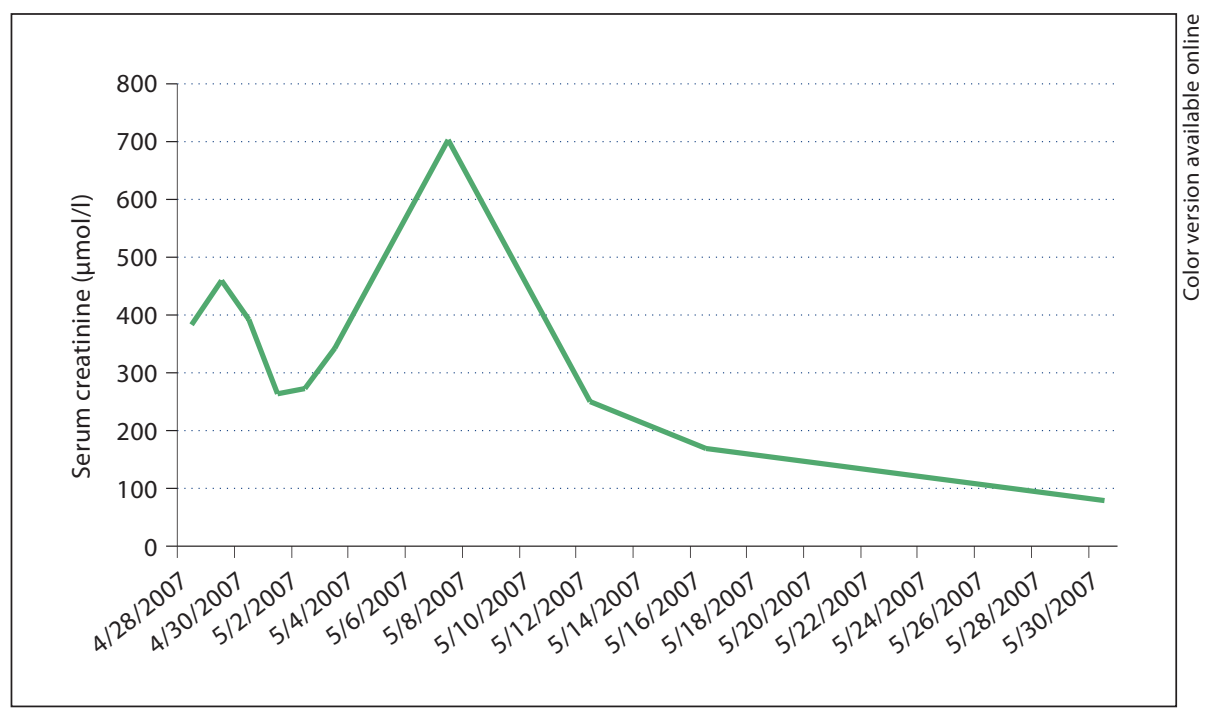

Fig. 2. Serum creatinine level,

can add insult to injury. That was the case at the initial presentation of our patient, who was taking simvastatin with many other medications. The dose of simvastatin was increased from 10 to $40 \mathrm{mg}$ daily, 10 days prior to this presentation. She developed severe diffuse muscular pain, urine discoloration and poor urinary output. The diagnosis of rhabdomyolysis, complicated by acute renal injury, hyperkalemia, hyponatremia, hypocalcemia, and hyperphosphatemia, was established.

Simvastatin was thought to be the culprit of this severe complication. It was stopped immediately after her admission. The patient was urgently started on hemodialysis, requiring a total of five sessions, with successful clinical outcome. Most of her laboratory findings, especially serum CK, potassium, sodium, calcium and phosphate levels, normalized within 10 days of treatment. Her kidney function worsened initially despite treatment. It took the full five sessions of hemodialysis to see a steady improvement in renal function. It fully recuperated within 1 month of the initial insult.

Our patient did present several features putting her at a higher risk for rhabdomyolysis. She was a thin female, relatively old, with a long-standing history of diabetes mellitus. What is interesting about her presentation though is the fact that she had been on simvastatin for 1 year prior to this event. It is the increase of the statin dose that led to the cascade of rhabdomyolysis, acute renal failure and electrolyte derangements. The comprehensive history and physical done for our patient, followed by the exactly-needed laboratory work, were key to establishing a precise diagnosis, followed by a successful treatment.

\section{Conclusion}

Rhabdomyolysis remains a dreadful condition for both patients and physicians. Indeed, its symptoms of severe myalgia, urine discoloration, decreased urine output, acute renal failure, and other complications are not easily forgotten by any patient who experiences this potentially fatal condition. Physicians in general are challenged in making the exact diagnosis, then by the treatment of this condition and its potential complications. This is more pertinent for doctors who will probably deal directly with it at the emergency room, outpatient clinic, inpatient setting, or in the dialysis unit.

Acute renal injury secondary to rhabdomyolysis is well established. It is usually associated with potentially dangerous metabolic and mineral derangements. Hemodialysis is usually needed to treat the renal insult and some of the dangerous electrolyte abnormalities.

Simvastatin, among other HMG-CoA reductase inhibitors, is known to cause rhabdomyolysis. Physicians should always keep in mind the possibility of this complication occurring, while either starting their patients on statins for the first time, or while attempting to increase the dose of a previously prescribed one. This statin side-effect alertness should be even higher when doctors are treating frail elderly patients with other co-morbid conditions, like diabetes mellitus, and polypharmacy. 


\section{References}

1 www.FDA.gov.

2 Sandy Craig, MD: Associate Program Director, Adjunct Assistant Professor, Department of Emergency Medicine, University of North Carolina at Chapel Hill, Carolinas Medical Center: Rhabdomyolysis. EMedicine.com.November 30, 2006.

3 Sauret JM, Marinides G, Wang GK: Rhabdomyolysis. Am Fam Physician 2002;65:907912.

4 Tomlinson SS, Mangione KK: Potential adverse effects of statins on muscle. Phys Ther 2005;85:459-465

$\checkmark 5$ Koya S, Crenshaw D, Agarwal A: Rhabdomyolysis and acuter renal failure after fire ant bites. J Gen Inten Med 2007;22:145-147.

6 Sorokin AV, Duncan B, Panetta R, Thompson PD: Rhabdomyolysis associated with pomegranate juice consumption. Am J Cardiol 2006;89:705-706.

7 Al-Shohaib S, Shaban A, Safwat M: Reversible acute renal failure due to rhabdomyolysis caused by lightning. Saudi J Kidney Dis Transpl Bull 1993;4:33-36.

$>8$ Wooltorton E: Rosuvastatin (Crestor) and rhabdomyolysis. CMAJ 2004;171:129.

-9 Graham DJ, Staffa JA, Shatin D, Andrade SE, Schech SD, La Grenade L, Gurwitz JH, Chan KA, Goodman MJ, Platt R: Incidence of hospitalized rhabdomyolysis in patients treated with lipid-lowering drugs. JAMA 2004;292: 2585-2590.

10 Brewer HB: Benefit-risk assessment of Rosuvastatin 10 to $40 \mathrm{mg}$. Am J Cardiol 2003;92: $23 \mathrm{~K}-29 \mathrm{~K}$.
11 Scott LJ, Curran MP, Figgitt DM: Rosuvastatin: a review of its use in the management of dyslipidemia. Am J Cardiovasc Drugs 2004;4:117-138

12 Zipes DP, Zvaifler NJ, Glassock RJ, Gilman S, Munoz A, Gogolak V, Gordis L, Dedon PC, Guengerich FP, Wasserman SA, Wiltzum JL, Wogan GN: Rosuvastatin: an independent analysis of risks and benefits. Med Gen Med 2006;8:73.

13 Middleton A, Binbrek AS, Fonseca FA, Wilpshaar W, Watkins C, Strandberg TE: Achieving 2003 European lipid goals with rosuvastatin and comparator statins in 6,743 patients in real-life clinical practice: DISCOVERY meta-analysis. Curr Med Res Opin 2006;22:1181-1191.

14 Davidson MH: Rosuvastatin safety: lessons from the FDA review and post-approval surveillance. Expert Opin Drug Saf 2004;3:547557.

15 Shepherd J, Hunninghake DB, Stein EA, Kastelein JJ, Harris S, Pears J, Hutchinson HG: Safety of rosuvastatin. Am J Cardiol 2004;94:882-888.

16 McKenney JM: Efficacy and safety of rosuvastatin in treatment of dyslipidemia. Am J Health Syst Pharm 2005;62:1033-1047.

17 Kasliwal R, Wilton LV, Cornelius V, AurichBarrera B, Shakir SA: Safety profile of rosuvastatin: results of a prescription-event monitoring study of 11,680 patients.

18 Culhane NS, Lettieri SL, Skae JR: Rosuvastatin for the treatment of hypercholesterolemia. Pharmacotherapy 2005;25:990-1000.

19 Lopez LM: Rosuvastatin: a high-potency HMG-CoA reductase inhibitor. J Am Pharm Assoc (2003) 2005;45:503-513.
20 Gruer PJ, Vega JM, Mercuri MF, Dobrinska MR, Tobert JA: Concomitant use of cytochrome P450 3A4 inhibitors and simvastatin. Am J Cardiol 1999;84:811-815.

21 Al Shoahib S: Simvastatin-induced rhabdomyolysis in a patient with chronic renal failure. Am J Nephrol 2000;20:212-213.

22 Pedersen TR, Tobert JA: Simvastatin: a review. Expert Opin Pharmacother 2004;5: 2583-2596.

23 Ho MR, Baker JE, Miller AL: Simvastatininduced rhabdomyolysis. Am J Emerg Med 2004;22:234-235

24 Federman DG, Hussain F, Walters AB: Fatal rhabdomyolysis caused by lipid-lowering therapy. South Med J 2001;94:1023-1026.

25 Shaukat A, Benekli M, Vladutiu GD, Slack JL, Wetzler M, Baer MR: Simvastatin-fluconazole causing rhabdomyolysis. Ann Pharmacother 2003;37:1032-1035.

26 Lee AJ, Maddix DS: Rhabdomyolysis secondary to a drug interaction between simvastatin and clarithromycin. Ann Pharmacother 2001;35:26-31.

-27 Roten L, Schoenenberger RA, Krähenbühl S, Schlienger RG: Rhabdomyolysis in association with simvastatin and amiodarone. Ann Pharmacother 2004;38:978-981.

28 Law M, Rudnicka AR: Statin safety: a systematic review. Am J Cardiol 2006;97:52C$60 \mathrm{C}$.

29 Ronaldson KJ, O'Shea JM, Boyd IW: Risk factors for rhabdomyolysis with simvastatin and atorvastatin. Drug Saf 2006;29:10611067.

30 www.Pubmed.gov. 